\title{
Caspase-8 activity has an essential role in CD95/Fas-mediated MAPK activation
}

\author{
AMM Kober ${ }^{1,6}$, S Legewie ${ }^{2,3,4,6}$, C Pforr ${ }^{1}$, N Fricker ${ }^{1,2}$, R Eils ${ }^{2,4,5,7}$, PH Krammer ${ }^{1,7}$ and IN Lavrik ${ }^{\star, 1,2,7}$
}

Stimulation of CD95/Fas/APO-1 results in the induction of both apoptotic and non-apoptotic signaling pathways. The processes regulating these two opposing pathways have not been thoroughly elucidated to date. In this study, using quantitative immunoblots, imaging, and mathematical modeling, we addressed the dynamics of the DED proteins of the death-inducing signaling complex (DISC), procaspase-8, and cellular FLICE inhibitory proteins (c-FLIPs) to the onset of CD95-mediated ERK1/2 and p38 mitogen-activated protein kinase (MAPK) activation. We found that CD95 DISC-induced caspase-8 activity is important for the initiation of ERK1/2 and p38 MAPK activation. The long c-FLIP isoform, c-FLIP ${ }_{L}$, and the short c-FLIP isoform, c-FLIP, inhibited MAPK induction by blocking caspase-8 processing at the DISC. Furthermore, we built a mathematical model describing CD95 DISC-mediated MAPK activation and apoptosis. The model quantitatively defined the dynamics of DED proteins, procaspase-8, and c-FLIP, which lead to caspase-8 activation and induction of apoptotic and non-apoptotic signaling pathways. In conclusion, the combination of biochemical analysis with mathematical modeling provides evidence for an important role of caspase-8 in CD95-mediated activation of MAPKs, while C-FLIP exerts a regulatory function in this process.

Cell Death and Disease (2011) 2, e212; doi:10.1038/cddis.2011.93; published online 6 October 2011

Subject Category: Experimental Medicine

CD95 (APO-1/Fas) ${ }^{1}$ is a member of the death receptor (DR) family. Stimulation of CD95 with its natural ligand CD95L or with agonistic anti-CD95 antibodies, such as anti-APO-1, induces apoptosis in sensitive cells. ${ }^{1}$ Stimulation of CD95 has also been reported to induce non-apoptotic pathways, such as $\mathrm{NF}-\kappa \mathrm{B}, \mathrm{Akt}$, ERK, and others. ${ }^{2}$ The death-inducing signaling complex (DISC) is formed within seconds after CD95 stimulation. ${ }^{3}$ The DISC comprises oligomerized CD95, the adaptor protein FADD, two isoforms of procaspase-8 (procaspase-8a and procaspase-8b), procaspase-10, and cellular FLICE inhibitory proteins (c-FLIPs). ${ }^{1,4-6}$

After binding to the DISC, procaspase-8a/b (p55/p53) homodimers undergo a conformational change and autocatalytic processing that results in the generation of active caspase-8. ${ }^{1,7}$ Procaspase-8a/b processing at the DISC involves cleavage at several Asp (D) residues between the prodomain and the small and large catalytic subunits (Supplementary Figure S1A). This results in the formation of the $\mathrm{N}$-terminal cleavage products $\mathrm{p} 43 / \mathrm{p} 41$, the prodomains p26/p24, as well as the C-terminal cleavage products p30, $\mathrm{p} 18$, and $\mathrm{p} 10 .^{8,9}$ It was proposed that the intrinsic catalytic activity of procaspase- 8 and active caspase- 8 bear highly restricted substrate repertoires. The catalytic activity of procaspase-8 was shown to be limited to procaspase-8 itself and c-FLIP. ${ }^{7,8,10}$ In contrast, mature caspase-8 does not cleave procaspase- 8 molecules, but efficiently processes effector procaspases, thus triggering the apoptotic signal. ${ }^{11}$ These different substrate specificities might in part contribute to the regulation of apoptosis and play a role in triggering either apoptotic or non-apoptotic CD95 signaling cascades. $^{8}$

c-FLIP is a well-described inhibitor of DR-mediated apoptosis. Three c-FLIP isoforms and two cleavage products have been characterized so far. ${ }^{12-15}$ The three c-FLIP isoforms include: long (L), short $(S)$, and Raji $(R)$, that is, c-FLIP $\mathrm{L}_{\mathrm{L}}, \mathrm{c}-\mathrm{FLIP}_{\mathrm{S}}$, and $\mathrm{C}-\mathrm{FLIP}_{\mathrm{R}}$, respectively (Supplementary Figure S1B). All three isoforms possess two DED domains and are therewith capable of binding to the DISC, where they competitively inhibit caspase-8 recruitment. Heterodimer formation of procaspase- 8 and c-FLIP at the DISC results in procaspase-8 activation and in procaspase-8-mediated cleavage of c-FLIP. In addition to their antiapoptotic role in DR-induced apoptosis, c-FLIP proteins and their cleavage products, p43-FLIP and p22-FLIP, were demonstrated to play a prominent role in the engagement of $\mathrm{NF}-\kappa \mathrm{B}$ signaling (Supplementary Figure S1B). 1,14,16

\footnotetext{
${ }^{1}$ Division of Immunogenetics, German Cancer Research Center (DKFZ), 69120 Heidelberg, Germany; ${ }^{2}$ Bioquant, 69120 Heidelberg, Germany; ${ }^{3}$ Institute of Molecular Biology, Mainz, Germany; ${ }^{4}$ Division of Theoretical Bioinformatics, German Cancer Research Center (DKFZ), 69120 Heidelberg, Germany and ${ }^{5}$ Department for Bioinformatics and Functional Genomics, Institute for Pharmacy and Molecular Biotechnology, University of Heidelberg, 69120 Heidelberg, Germany

${ }^{*}$ Corresponding author: IN Lavrik, Division of Immunogenetics, German Cancer Research Center (DKFZ), Im Neuenheimer Feld 280, Heidelberg 69120, Germany. Tel: + 49622142 3765; Fax: + 49622141 1715; E-mail: i.lavrik@dkfz.de

${ }^{6}$ These authors are co-first authors.

${ }^{7}$ These authors are co-last authors.

Keywords: CD95; caspase-8; modeling; C-FLIP; MAPK

Abbreviations: DISC, death-inducing signaling complex; c-FLIPs, cellular FLICE inhibitory proteins; MAPKs, mitogen-activated protein kinases; c-FLIP ${ }_{L}, \mathrm{C}-\mathrm{FLIP}$ long; $c-$ FLIP $_{S}$, c-FLIP short; c-FLIP, c-FLIP Raji; HeLa-CD95, HeLa cells with CD95 overexpression; HeLa-CD95- $F_{R}$, HeLa-CD95 cells with c-FLIP ${ }_{R}$ overexpression; HeLa-CD95- $\mathrm{F}_{\mathrm{L}}$, HeLa-CD95 cells with c-FLIP ${ }_{\mathrm{L}}$ overexpression; HeLa-CD95-C8 dr, HeLa-CD95 cells with caspase-8 downregulation; HeLa-CD95-F dr, HeLa-CD95 cells with C-FLIP downregulation

Received 11.7.11; revised 01.8.11; accepted 04.8.11; Edited by J Silke
} 
Recently, it has been reported that CD95L is not only a potent inducer of apoptosis, but can also activate multiple non-apoptotic pathways, in particular induction of mitogenactivated protein kinases (MAPKs). ${ }^{2,17-21}$ MAPKs are implicated in growth, proliferation, differentiation, migration, and apoptosis. It has been reported that activation of the ERK MAPK pathway upon CD95 crosslinking has a functional implication in nerve recovery ${ }^{22}$ and prevention of CD95-induced apoptosis. ${ }^{23}$ In addition, CD95 signaling was shown to activate p38 MAPK. ${ }^{24}$ Although caspase activity has been reported to be involved in CD95-mediated MAPK activation in previous studies, ${ }^{17,21,24}$ the distinct mechanisms of ERK and p38 MAPK activation following CD95 triggering remain elusive.

Studies of CD95 signaling and apoptosis using systems biology have recently provided significant insights into quantitative regulation of these processes. A number of models describe the regulation of caspase activation, ${ }^{25,26}$ the intrinsic pathway, ${ }^{27}$ and the extrinsic pathway regulation. ${ }^{28-30}$ In addition, a model of the interplay between CD95-mediated apoptosis and NF- $\kappa \mathrm{B}$ induction has been reported recently. ${ }^{16}$ Nevertheless, a model describing the crosstalk between CD95-mediated apoptosis and MAPK activation has still been missing.

The aim of this study is to provide new insights into the mechanisms at the DISC, leading to the activation of MAPKs upon stimulation of CD95, in particular to delineate the role of procaspase- 8 and c-FLIP isoforms in this process. Activation of the MAPK family members, ERK1/2 and p38, upon CD95 stimulation was quantitatively analyzed using HeLa cell lines overexpressing CD95 (HeLa-CD95 cells) ${ }^{16,30}$ with different expression levels of c-FLIP isoforms and procaspase-8. Experimental data supported by mathematical modeling demonstrated that the degree of procaspase-8 activation at the DISC, which is regulated by c-FLIP proteins, is directly linked to the degree of CD95-mediated induction of MAPKs, highlighting the crucial role of caspase- 8 activity in this signaling pathway.

\section{Results}

CD95 stimulation leads to a dose-dependent MAPK activation in HeLa-CD95 cells. To understand the mechanisms of CD95-mediated MAPK activation in mechanistic and quantitative terms, we utilized HeLa-CD95 cell lines that have been established in our lab. ${ }^{16,30}$ These are the only available cell lines, in which the precise concentrations of the major apoptotic modulators have been defined. The exact number of the major DISC components, that is, procaspase-8, c-FLIP, FADD, and CD95, in these cell lines as well as quantitative responses with respect to CD95-mediated apoptosis and $\mathrm{NF}-\kappa \mathrm{B}$ induction have been determined in our previous work. ${ }^{16,30}$ Accordingly, these cell lines constitute an ideal tool for further investigation of the initial events of CD95-mediated MAPK activation. Furthermore, we have generated HeLa-CD95 cells with different levels of c-FLIP and procaspase- 8 by downregulation and overexpression of these proteins. ${ }^{16,30}$ Our panel of HeLa-CD95 cells comprised: HeLa-CD95 cells with c-FLIP ${ }_{R}$ overexpression (HeLa-CD95- $F_{R}$ ), HeLa-CD95 cells with $C-F L I P_{L}$ overexpression (HeLa-CD95- $F_{L}$ ), HeLaCD95 cells with caspase-8 downregulation (HeLa-CD95-C8 dr), and HeLa-CD95 cells with c-FLIP downregulation (HeLaCD95-F dr).

Upon stimulation with agonistic anti-APO-1 antibodies, HeLa-CD95 cells underwent apoptosis as observed by morphological changes characteristic for apoptotic cell death in live cell imaging (Figure 1). In accordance with previous reports, overexpression of c-FLIP isoforms and downregulation of caspase- 8 decreased the rates of apoptosis (Figure 1b). ${ }^{16,30}$

To characterize CD95-mediated activation of MAPKs, HeLa-CD95 cells were treated with either 250 or $1500 \mathrm{ng} / \mathrm{ml}$ anti-APO-1. Immunoblot (IB) analysis of phosphorylated ERK1/2 and p38 showed that these MAPKs were highly activated upon stimulation of CD95 with anti-APO-1 (Figure 2). The onset of activation was found to be stimulus-dependent for both ERK1/2 and p38. ERK1/2 activation first peaked at $90 \mathrm{~min}$ for a stimulus of $250 \mathrm{ng} / \mathrm{ml}$ anti-APO-1, whereas stimulation with $1500 \mathrm{ng} / \mathrm{ml}$ anti-APO-1 led to a first peak of ERK1/2 activation already at 60 min (Figure 2a). Remarkably, ERK $1 / 2$ activation subsequently decreased before it reached a second peak at about $180 \mathrm{~min}$ for a stimulus of $250 \mathrm{ng} / \mathrm{ml}$ anti-APO-1 and at $120 \mathrm{~min}$ for stimulation with $1500 \mathrm{ng} / \mathrm{ml}$ anti-APO-1 (Figure 2a). Similar to ERK1/2, p38 also showed an earlier detectable onset of activation with the higher stimulus (Figure 2b). In contrast to the biphasic activation of ERK1/2, activation of p38 steadily increased with time (Figure 2b).

The phosphorylation of MAPKs was accompanied by CD95-triggered cleavage of $\mathrm{C}-\mathrm{FLIP}_{\mathrm{L}}$ into $\mathrm{p} 43-\mathrm{FLIP}$ and procaspase-8a/b processing into $\mathrm{p} 43 / \mathrm{p} 41, \mathrm{p} 30$, and $\mathrm{p} 18$ (Figure 2). The appearance of cleavage products was found to be stimulus-dependent in accordance with previous reports: ${ }^{9,30} \mathrm{c}-\mathrm{FLIP}_{\mathrm{L}}$ and procaspase-8a/b cleavage products appeared earlier upon stimulation of CD95 with $1500 \mathrm{ng} / \mathrm{ml}$ anti-APO- 1 than with $250 \mathrm{ng} / \mathrm{ml}$ anti-APO-1. These data show that CD95 stimulation with anti-APO-1 induces the activation of ERK1/2 and p38 MAPKs in a dose-dependent manner, which, in turn, directly correlates with kinetics of procaspase-8 cleavage.

c-FLIP proteins inhibit CD95-mediated MAPK activation. c-FLIP proteins have been reported to be important regulators of CD95-mediated apoptosis and $\mathrm{NF}-\kappa \mathrm{B}$ induction. ${ }^{16}$ To determine whether c-FLIP proteins play a regulatory role in CD95-mediated activation of MAPKs, we used HeLa-CD95 cell lines with stable overexpression or downregulation of $c-F L I P$ isoforms.

Overexpression of $c-$ FLIP $_{R}$ or C-FLIP $\mathrm{P}_{\mathrm{L}}$ in HeLa-CD95 cells reduced the activation of MAPKs upon CD95-stimulation (Figure 3 and Supplementary Figures S2 and S3). A comparison between MAPK activation in HeLa-CD95 and HeLa-CD95- $F_{R}$ cells demonstrated that overexpression of c-FLIP $P_{R}$ strikingly reduced activation of both ERK1/2 and p38 (Figure 3 and Supplementary Figure S3). The amounts of phosphorylated ERK1/2 and p38 were quantified and normalized to tubulin, which served as a loading control. Alignments of time series from repeated experiments were performed to obtain comparability (Figures $3 b$ and $d$ ). Overexpression of 

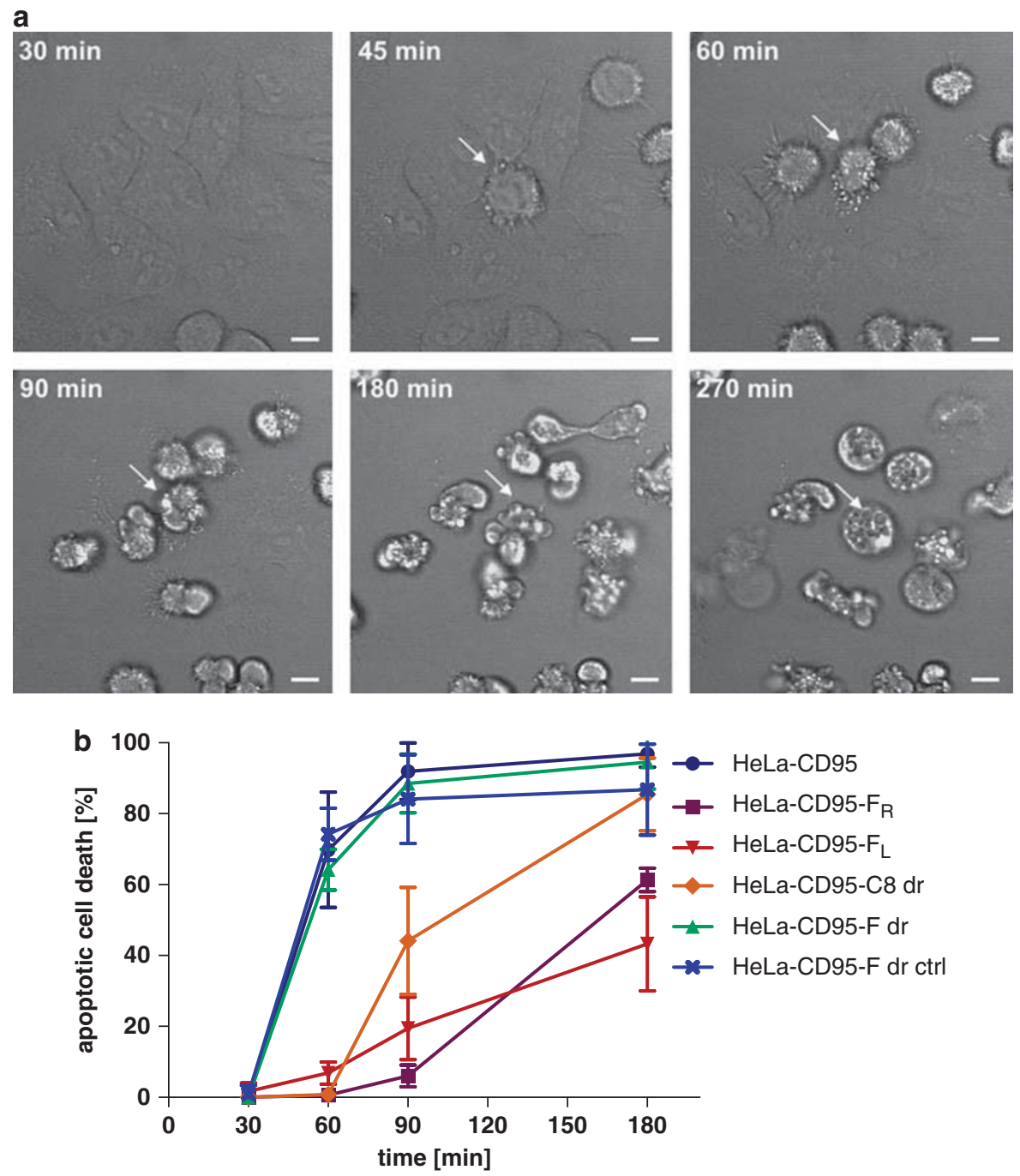

Figure 1 Apoptotic cell death of HeLa-CD95 cell lines upon stimulation with $1000 \mathrm{ng} / \mathrm{ml}$ anti-APO-1. Different HeLa-CD95 cell lines were stimulated with $1000 \mathrm{ng} / \mathrm{ml}$ antiAPO-1 and subjected to time-lapse microscopy. (a) Representative images for HeLa-CD95 cells are shown. Apoptosis was detected based on morphological characteristics associated with apoptotic cell death, including condensation of the cytoplasm, membrane blebbing, and formation of apoptotic bodies. White arrows indicate a cell that shows typical characteristics of apoptotic cell death, that is, condensation of the cytosol (45 and $60 \mathrm{~min}$ ), membrane blebbing (180 min), and formation of apoptotic bodies $(270 \mathrm{~min})$. (b) For the indicated points in time, the number of cells showing onset of apoptosis characterized by cytoplasm condensation and the total number of cells were counted to determine the percentage of apoptotic cell death. Displayed results are averaged values for five images per cell line and time. HeLa-CD95, HeLa cell line overexpressing CD95; HeLa-CD95- $\mathrm{F}_{\mathrm{R}}$, HeLa-CD95 cells with c-FLIP $\mathrm{R}_{\mathrm{R}}$ overexpression; HeLa-CD95- $\mathrm{F}_{\mathrm{L}}$, HeLa-CD95 cells with c-FLIPL overexpression; HeLa-CD95-C8 dr, HeLa-CD95 cells with caspase-8 downregulation; HeLa-CD95-F dr, HeLa-CD95 cells with c-FLIP downregulation; HeLa-CD95-F ctrl, corresponding control cell line to HeLaCD95- $\mathrm{F} \mathrm{dr}$

c-FLIP $\mathrm{R}_{\mathrm{R}}$ inhibited processing of procaspase-8 and $\mathrm{c}-\mathrm{FLIP} \mathrm{L}$, which is in accordance with previous publications on the inhibitory role of $\mathrm{c}-\mathrm{FLIP}_{\mathrm{R}}{ }^{13}$ Consistent with this observation, the procaspase-8a/b cleavage products $p 43 / p 41$ and p18 were clearly detectable upon stimulation in HeLaCD95 cell lysates, but not in HeLa-CD95- $F_{R}$ cell lysates (Figures $3 a$ and $\mathrm{c}$ ).

Comparable results were obtained for HeLa-CD95- $\mathrm{F}_{\mathrm{L}}$ cells. Overexpression of $C-F L I P L$ substantially reduced activation of ERK1/2 and p38 upon stimulation with anti-APO-1 (Supplementary Figure S2). These data demonstrate that overexpression of $\mathrm{C}-\mathrm{FLIP}_{\mathrm{R}}$ or $\mathrm{C}-\mathrm{FLIP}_{\mathrm{L}}$ reduced $\mathrm{CD}$ 95-mediated activation of MAPKs and resulted in incomplete processing of procaspase-8a/b.
To further analyze the role of c-FLIPs in CD95-mediated activation of MAPKs, an siRNA construct targeting c-FLIP was stably transfected into HeLa-CD95 cells to generate the HeLa-CD95-F dr cell line with downregulation of c-FLIP. The corresponding HeLa-CD95-F ctrl control cell line was generated by transfection of an empty vector control. Quantification of c-FLIP downregulation in the unstimulated case for all experiments averaged out at a downregulation by $68 \%$ in comparison to the control cell line. HeLa-CD95, HeLa-CD95-F $\mathrm{dr}$, and HeLa-CD95-F ctrl were stimulated with $1000 \mathrm{ng} / \mathrm{ml}$ anti-APO-1 for 15-120 min and total cellular lysates were analyzed by IB (Figure 4 and Supplementary Figure S4). Interestingly, C-FLIP downregulation was found to reduce CD95-mediated activation of ERK1/2 and p38 (Figure 4), 
a

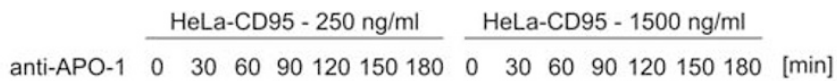

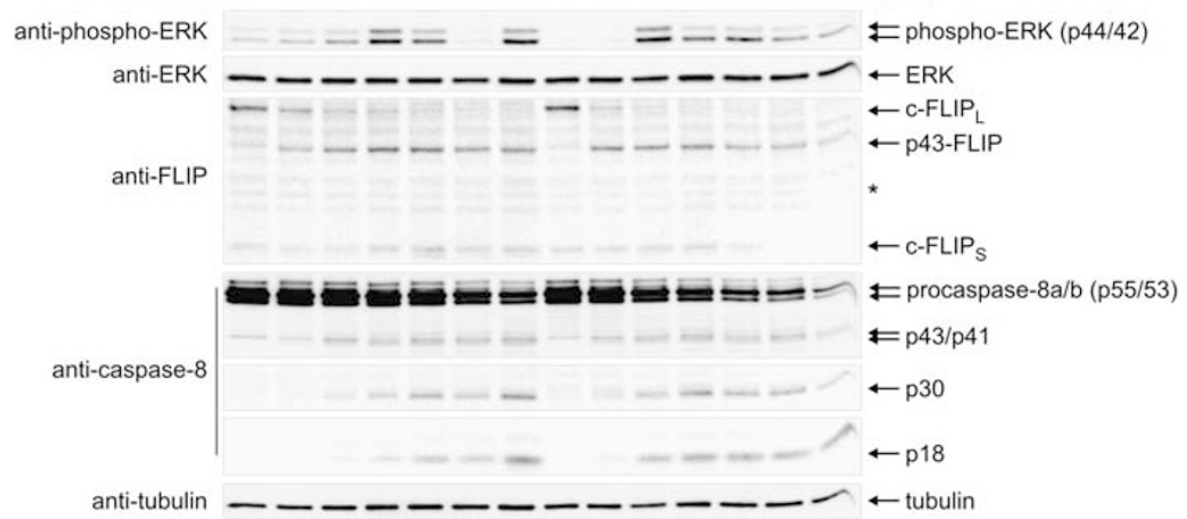

b

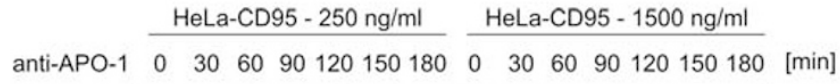

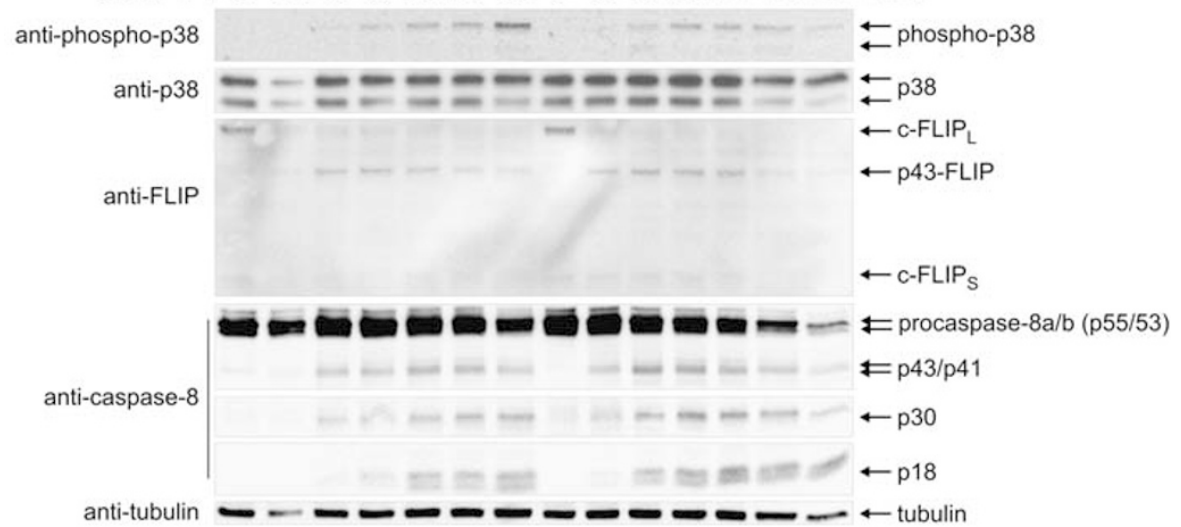

Figure 2 Anti-APO-1 concentration-dependent onset of ERK and p38 MAPK activation. HeLa-CD95 cells were stimulated with 250 or $1500 \mathrm{ng} / \mathrm{ml}$ anti-APO-1 for the indicated time periods. Total cellular lysates were analyzed by SDS-PAGE and IB using the indicated primary antibodies. Representative IB results for ERK (a) and p38 (b) activation are shown. Asterisks indicate nonspecific bands

albeit to a lesser extent than by overexpression of $c-F L I P L$ or $\quad c-F_{R}$. Remarkably, less caspase-8 cleavage product p18 was detectable upon c-FLIP downregulation (Figures $4 \mathrm{a}$ and $\mathrm{c}$ ).

According to these data, both overexpression and downregulation of c-FLIP reduce CD95-mediated activation of MAPKs and procaspase- $8 a / b$ processing. These findings indicate that C-FLIP exhibits a regulatory function with properties depending on its cellular concentration.

Caspase-8 plays the major role in CD95-mediated activation of MAPKs. The unifying characteristic inhibiting MAPK activation in so far studied conditions is a reduced processing of procaspase- 8 reflected by the lower amount of procaspase- $8 \mathrm{a} / \mathrm{b}$ cleavage products. Therefore, the role of procaspase- 8 and active caspase- 8 was further investigated. Treatment of HeLa-CD95 cells (Supplementary Figure S5) and HeLa-CD95 cells overexpressing C-FLIPL or C-FLIP $\mathrm{R}_{\mathrm{R}}$ (Supplementary Figure S6) with the pan-caspase inhibitor zVAD-fmk inhibited phosphorylation of ERK $1 / 2$ and p38. These data provided the first hint at the involvement of caspase activity in CD95-mediated MAPK activation in our system.
To analyze caspase-8 involvement in CD95-mediated activation of MAPKs more directly, the HeLa-CD95-C8 dr cell line with stable downregulation of caspase- 8 by RNA interference ${ }^{16}$ was used. HeLa-CD95 and HeLa-CD95-C8 dr cells were stimulated with $1000 \mathrm{ng} / \mathrm{ml}$ anti-APO-1 for time periods ranging between 15 and $180 \mathrm{~min}$. Downregulation of caspase-8 substantially reduced activation of ERK1/2 and p38 upon CD95 stimulation (Figure 5 and Supplementary Figure S7). Quantification of procaspase-8a/b downregulation in the unstimulated case for all experiments averaged out at a downregulation by $60 \%$. In contrast to HeLa-CD95 cell lysates, almost no active procaspase-8a/b cleavage products p43/p41, p30, and p18 were detectable in HeLa-CD95-C8 dr cell lysates. These data suggest that procaspase- 8 and its processing are required for CD95-mediated activation of MAPKs.

Modeling revealed that the ratio of procaspase-8 to c-FLIP at the DISC plays the crucial role in CD95mediated MAPK activation. Our experimental measurements suggested that MAPK activation occurs downstream of DISC formation and caspase-8 activation. To further confirm this hypothesis, we included caspase-8-mediated 

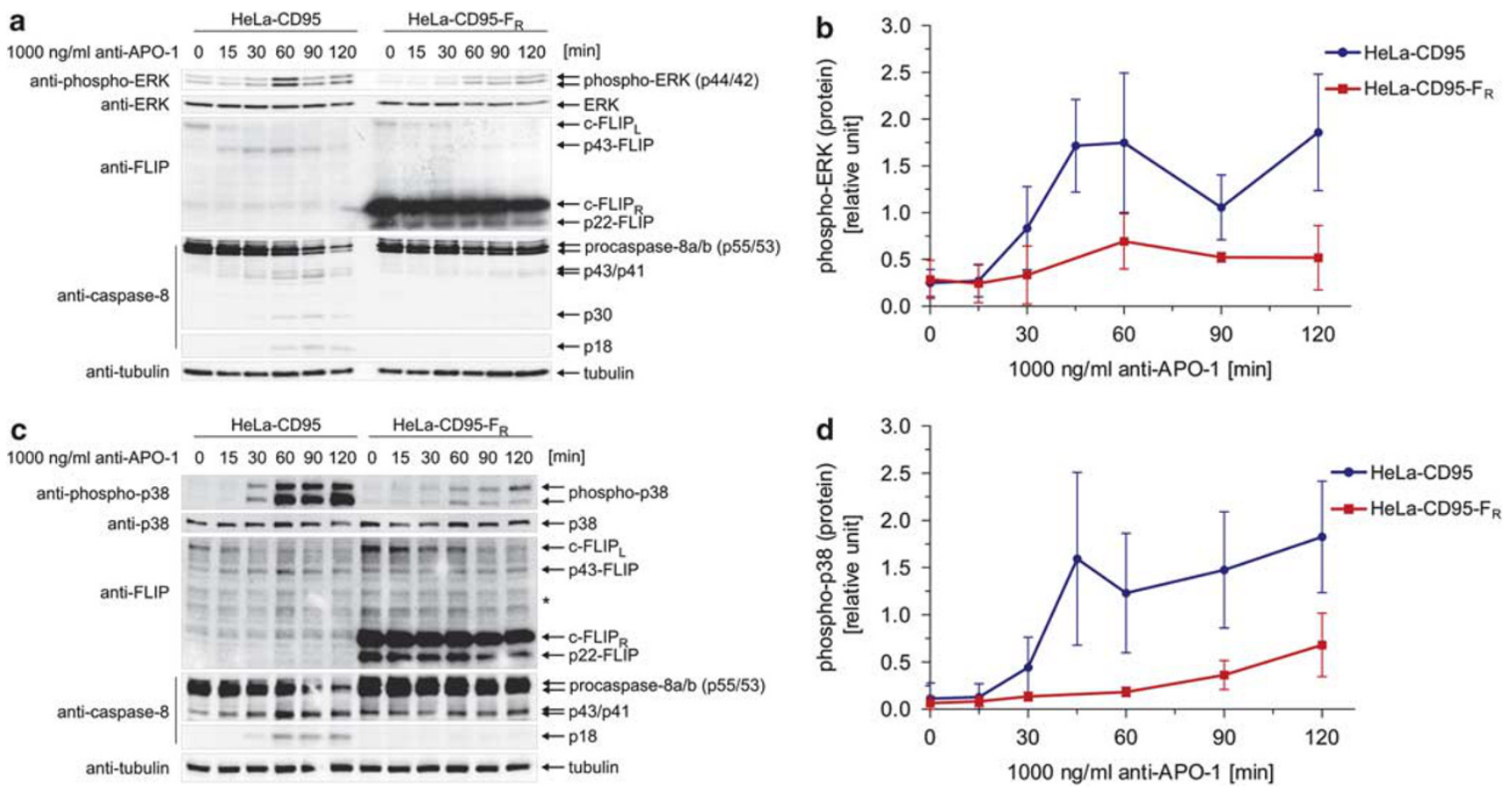

Figure 3 Reduced activation of ERK and p38 upon c-FLIP $\mathrm{R}_{\mathrm{R}}$ overexpression. HeLa-CD95 and HeLa-CD95- $\mathrm{F}_{\mathrm{R}}$ cells were stimulated with $1000 \mathrm{ng} / \mathrm{ml}$ anti-APO-1 for the indicated time periods. Total cellular lysates were analyzed by SDS-PAGE and IB using the indicated primary antibodies. Representative IB results for ERK (a) and p38 (c) activation as well as the averaged quantified signals of scaled time courses with standard deviations of all performed experiments (b and $\mathbf{d})$ are shown. Displayed results are representative of two $(0-120 \mathrm{~min})$ and additional three $(E R K)$ or two (p38) $(0-60 \mathrm{~min})$ independent experiments. HeLa-CD95- $F_{\mathrm{R}}$, HeLa-CD95 cells with c-FLIP $\mathrm{R}_{\mathrm{R}}$ overexpression. Asterisks indicate nonspecific bands. Independent repeats of these experiments are shown in Supplementary Figure S3

\section{a} $1000 \mathrm{ng} / \mathrm{ml}$ anti-APO-1 0 HeLa-CD95 HeLa-CD95-F ctrl HeLa-CD95-F dr 1030456090120030456090120030456090120 [min]
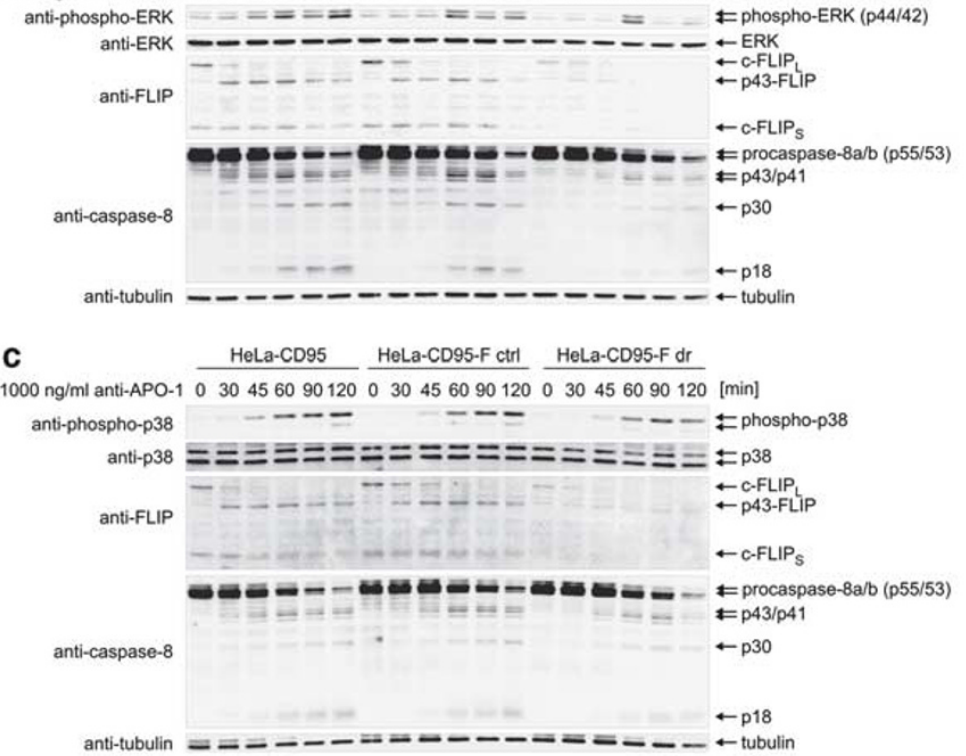
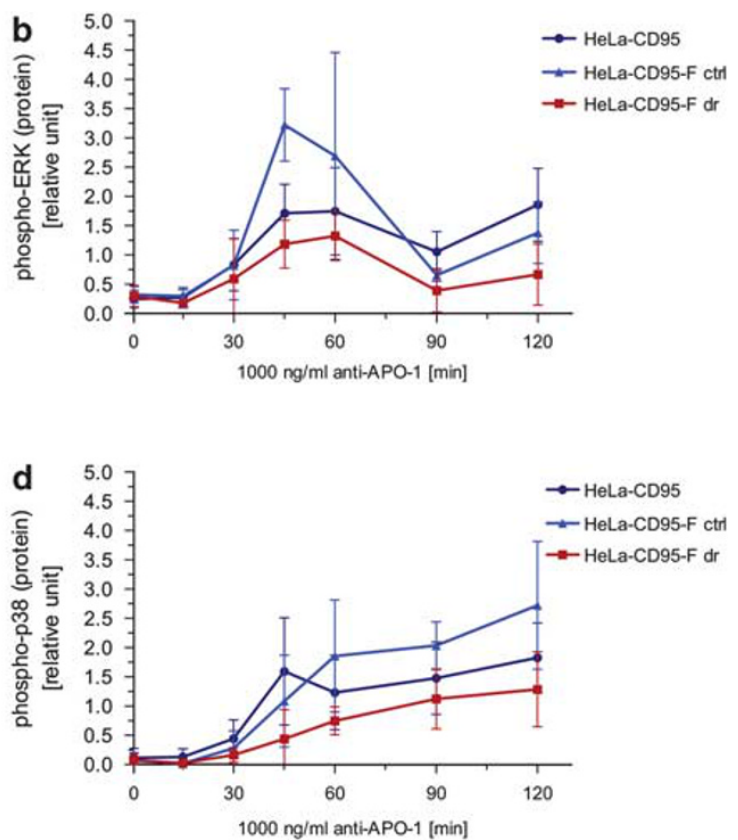

Figure 4 Reduced activation of ERK and p38 upon c-FLIP downregulation. HeLa-CD95, HeLa-CD95-F ctrl, and HeLa-CD95-F dr cells were stimulated with 1000 ng/ml anti-APO-1 for the indicated time periods. Total cellular lysates were analyzed by SDS-PAGE and IB using the indicated primary antibodies. Representative IB results for ERK (a) and p38 (c) activation as well as the averaged quantified signals of scaled time courses with standard deviations of all performed experiments (b and d) are shown. Displayed results are representative for four independent experiments. HeLa-CD95-F dr, HeLa-CD95 cells with c-FLIP downregulation; HeLa-CD95-F ctrl, control cell line without c-FLIP downregulation. Independent repeats of these experiments are shown in Supplementary Figure S4 

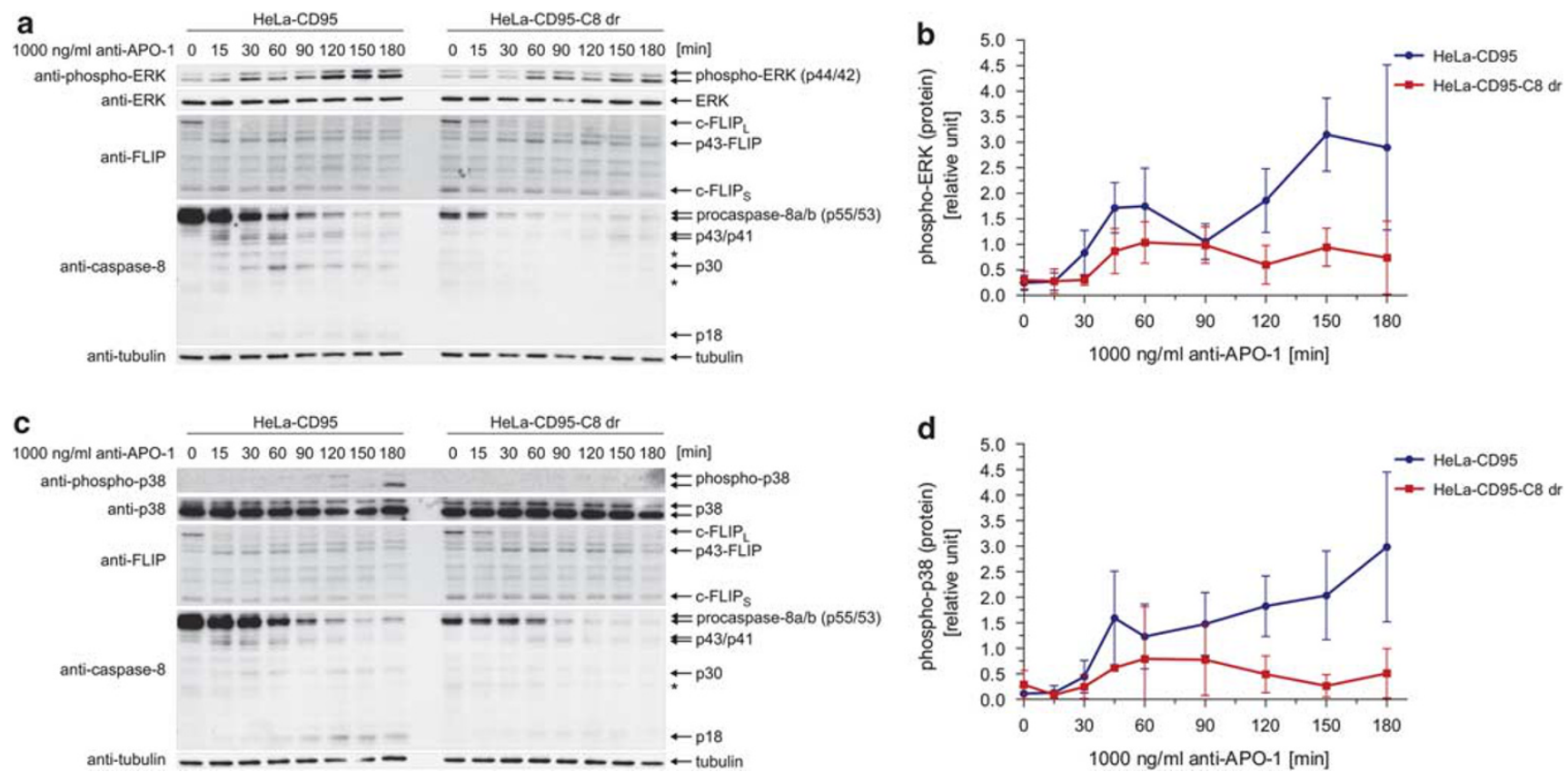

Figure 5 Reduced activation of ERK and p38 upon caspase-8 downregulation. HeLa-CD95 and HeLa-CD95-C8 dr cells were stimulated with $1000 \mathrm{ng} / \mathrm{ml}$ anti-APO-1 for the indicated time periods. Total cellular lysates were analyzed by SDS-PAGE and IB using the indicated primary antibodies. Representative IB results for ERK (a) and p38 (c) activation as well as the averaged quantified signals of scaled time courses with standard deviations of all performed experiments (b and $\mathbf{d})$ are shown. Displayed results are representative for three (0-180 $\mathrm{min})$ and additional two (0-120 min) independent experiments. HeLa-CD95-C8 dr, HeLa-CD95 cells with caspase-8 downregulation. Asterisks indicate nonspecific bands. Independent repeats of these experiments are shown in Supplementary Figure S7

MAPK activation in our previously published mathematical mode $^{30}$ and compared the model trajectories to experimental time-course data. For simplicity, the analysis was restricted to p38 activation. In the model, p38 phosphorylation was assumed to be directly activated by enzymatically active caspase-8 (Supplementary Figure S8). In addition, we considered a p38 dephosphorylation step in the model and assumed it to be not regulated by caspase- 8 . The ordinary differential equations of the model can be found in the Supplementary Material.

Our previously published mode ${ }^{30}$ was based on timecourse measurements in HeLa-CD95 cells stimulated with CD95L, while agonistic anti-APO-1 antibodies were used as the stimulus in this work. Moreover, the present data set includes a novel experimental condition that was lacking in the calibration of the previous model (i.e., caspase-8 downregulation). We, therefore, derived an improved model by simultaneously fitting all kinetic parameters to the anti-APO-1 and CD95L data sets. Specifically, we assumed that CD95L and anti-APO-1 stimulation conditions solely differ in the biologically active ligand concentration and the ligand binding rate, whereas the kinetic parameters of intracellular reactions are the same for both conditions (see Supplementary Material for fitted parameters). The trajectories of the best-fit model lie within the experimental error (Figure $6 \mathrm{a}$ and Supplementary Figure S9). This further supports our hypothesis that p38 activation occurs downstream of caspase-8 activity.

The model could reproduce our experimental observation that c-FLIP downregulation and overexpression both block procaspase- 8 processing and p38 activation in response to anti-APO-1 stimulation (Figure 6). However, in our previous work, we observed that c-FLIP downregulation does not significantly affect procaspase-8 processing upon CD95L stimulation of HeLa-CD95 cells. ${ }^{30}$ The model explains these different observations upon c-FLIP downregulation by a ligand-specific effect considering that the activity of antiAPO-1 is lower than the activity of CD95L. As procaspase- 8 is processed more rapidly in C-FLIP $\mathrm{P}_{\mathrm{L}}$-procaspase-8 heterodimers than in procaspase- 8 homodimers, ${ }^{30} \mathrm{C}-\mathrm{FLIP}$ downregulation can possibly reduce procaspase- 8 activation. The absolute concentrations of c-FLIP proteins (each $\sim 500$ molecules per cell) in HeLa-CD95 cells are much lower than those of CD95 (150 000 molecules per cell) and procaspase-8 (250 000 molecules per cell). ${ }^{30}$ Given that c-FLIP binds to the CD95 DISC with much higher affinity than its by far more abundant competitor procaspase-8, and that in our model, anti-APO-1 activates slightly less than half of the CD95 pool, while CD95L activates the whole receptor pool, the contribution of c-FLIP heterodimers to procaspase-8 processing are more pronounced upon anti-APO-1 stimulation when compared to CD95L.

Caspase-8 activity is essential to MAPK activation in response to anti-APO-1 stimulation (see above). Formation of $p 18$ occurs at the DISC by processing of p43/p41 and is modulated by c-FLIP. We, therefore, hypothesized that $\mathrm{p} 18$ formation and MAPK activation are controlled by the ratio of p43/p41 and p43-FLIP at the DISC. Thus, the relative concentrations of p43-FLIP and p43/p41 at the DISC were analyzed in silico (Figure 6b). The simulations reveal that the absolute concentrations of p43/p41 and p43-FLIP are poor predictors of $p 18$ formation: for instance, $c$-FLIP $P_{L}$ overexpression or C-FLIP downregulation hardly affect maximal DISC 
a
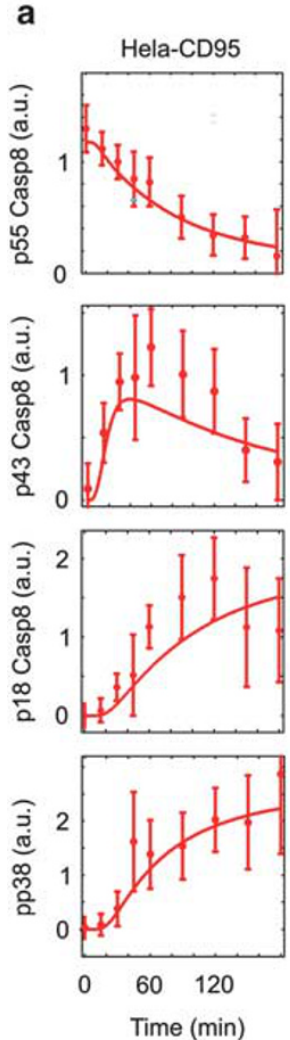
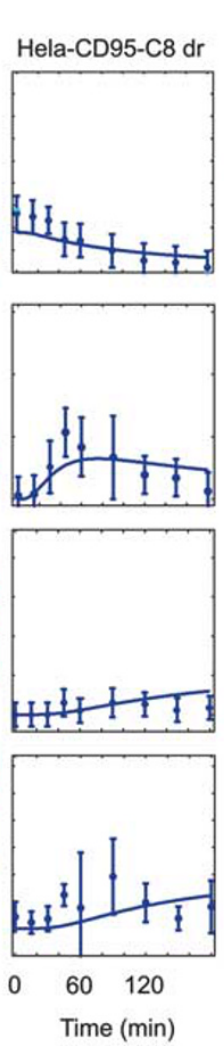

Model Fit
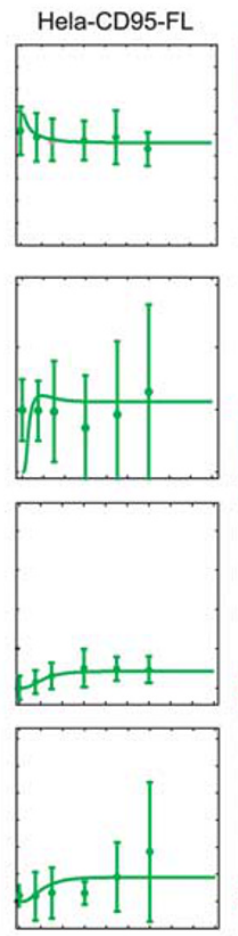

$\begin{array}{lc}60 & 120 \\ \text { Time (min) }\end{array}$
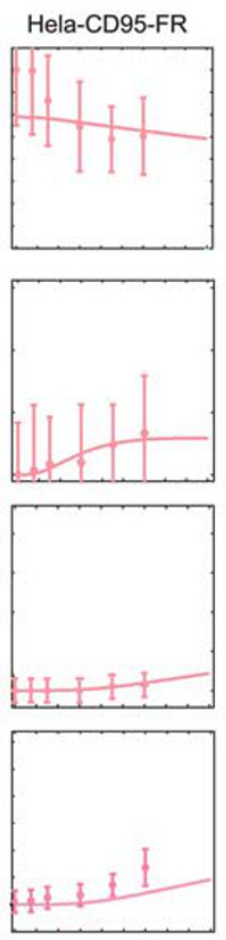

$0 \quad 60 \quad 120$

Time (min)
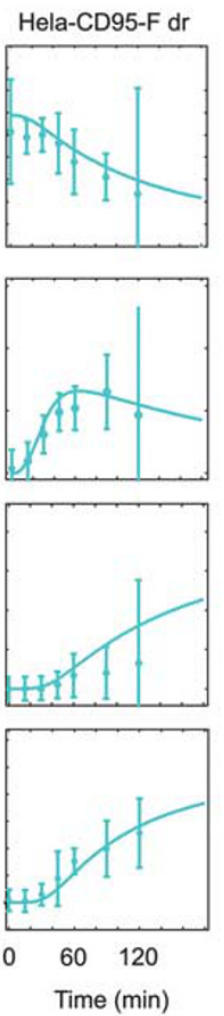

b
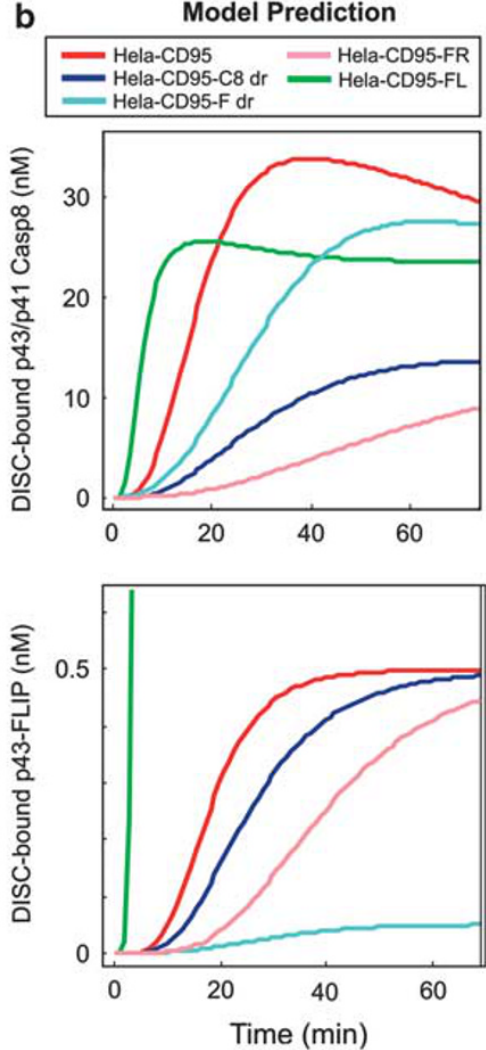

Figure 6 The best-fit model captures time courses of caspase-8/MAPK activation and predicts the dynamics of DISC formation. (a) Model fits to experimental measurements of p55/p53 procaspase-8, caspase-8 cleavage products p43/p41 and p18, as well as phospho-p38 (pp38) in response to stimulation with $1000 \mathrm{ng} / \mathrm{ml}$ anti-APO-1. Each column corresponds to measurements in one of the HeLa-CD95 cell lines (indicated on top), and each row corresponds to one molecular species (indicated on the left). All kinetic parameters of the model (except the ligand concentration and the ligand binding rate) were fitted globally to measurements in anti-APO-1 and CD95L-stimulated HeLa-CD95 cells (see Supplementary information for kinetic parameters and fits to CD95L data). (b) Predicted dynamics of p43/p41 and p43-FLIP recruitment to the DISC in response to stimulation with $1000 \mathrm{ng} / \mathrm{ml}$ anti-APO-1. Each color corresponds to one of the HeLa-CD95 cell lines as indicated in the legend

recruitment of procaspase-8 despite having significant impact on p18 formation (Figure 6b). Likewise, p43-FLIP generation at the DISC is essentially unchanged upon procaspase-8 downregulation or C-FLIP $\mathrm{R}_{\mathrm{R}}$ overexpression (Figure 6b). However, we note that all conditions with diminished $\mathrm{p} 18$ formation are characterized by a strong alteration in the p43-FLIP to p43/p41 ratio at the DISC (Figure 6b). This supports our hypothesis that the c-FLIP/procaspase- 8 ratio is the key to CD95-induced MAPK activation.

To verify that our model indeed captures the dynamics of protein recruitment and generation of cleavage products at the DISC, we analyzed DISC formation in HeLa-CD95 cells and in HeLa-CD95 cells upon downregulation of procaspase-8 (Figure 7 and Supplementary Figure S10). The model predicted that procaspase-8 downregulation delays p43-FLIP generation at the DISC, but does not affect its final level (Figure 6b). With a stimulus of $1000 \mathrm{ng} / \mathrm{ml}$ anti-APO-1, p43FLIP at the DISC indeed reached an early saturation level at around $20 \mathrm{~min}$ in HeLa-CD95 cells (Figures $7 \mathrm{a}$ and b). In contrast, the amount of p43-FLIP at the DISC continued to increase in HeLa-CD95-C8 dr cells within 60 min of stimulation with anti-APO-1. Moreover, after $20 \mathrm{~min}$ the level of p43-FLIP was observed to be lower in HeLa-CD95-C8 dr cells than in HeLa-CD95 cells. Taken together, the experiments confirm the decelerated p43-FLIP generation in
HeLa-CD95-C8 dr cells as predicted by the model. However, experimental data and the model slightly disagreed at late time points: the model suggested that the final level of p43-FLIP should be unaffected by caspase-8 downregulation, while the experimentally measured p43-FLIP reached a comparably higher level in HeLa-CD95-C8 dr cells after $60 \mathrm{~min}$. The model also predicted that the generation of p43/p41 at the DISC should be significantly decreased, at both early and late points of time (Figure $6 \mathrm{~b}$, top). This prediction agrees well with experimental data (Figure 7a). We conclude that our model captures the dynamics of protein recruitment to the DISC, and that the ratio of p43/p41 and p43-FLIP at the DISC is dramatically altered in cells with the downregulation of procaspase-8. These effects were found to be even more pronounced upon stimulation with the lower stimulus of $250 \mathrm{ng} / \mathrm{ml}$ anti-APO-1 (Figures 7c and d). The observation of an altered ratio of C-FLIP to procaspase-8 at the DISC further confirms the data obtained in the present work that demonstrate an essential role for C-FLIP proteins and procaspase-8 in CD95mediated activation of ERK1/2 and p38 MAPKs.

\section{Discussion}

The pivotal role of CD95 signaling in the induction of apoptosis is well established. Nevertheless, CD95 signaling is also 

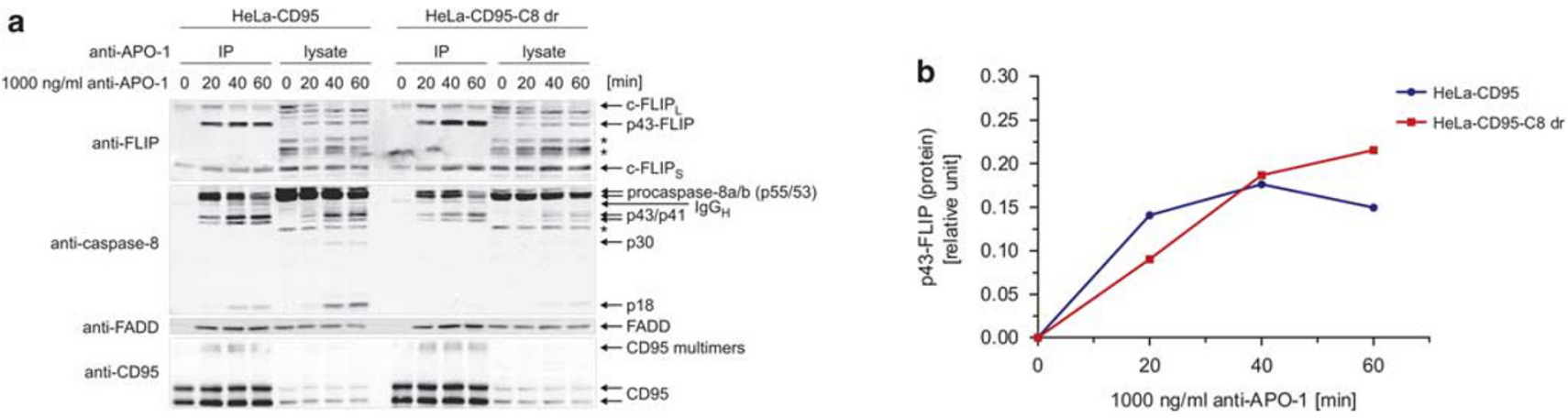

C
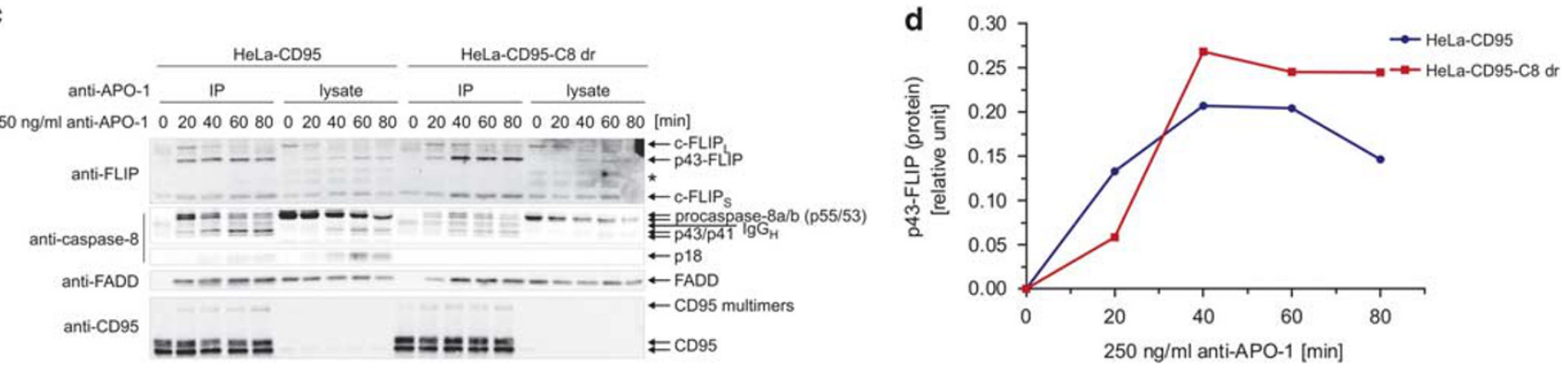

Figure 7 Altered ratio of c-FLIP/C8 at the DISC upon caspase-8 downregulation and altered kinetics with lower stimulus at the DISC. HeLa-CD95 cells and HeLa-CD95-C8 dr cells were treated with 1000 or $250 \mathrm{ng} / \mathrm{ml}$ anti-APO- 1 for the indicated time periods and IP of the DISC was performed. Total cellular lysates and IPs of the DISC were analyzed by SDS-PAGE and IB using the indicated primary antibodies. Representative IB results (a and $\mathbf{c}$ ) and the quantification for the displayed IB (b and $\mathbf{d})$ are shown. Displayed results are representative of two independent experiments. HeLa-CD95-C8 dr, HeLa-CD95 cells with caspase-8 downregulation. Asterisks indicate nonspecific bands. Independent repeats of these experiments are shown in Supplementary Figure S10

involved in mediating non-apoptotic pathways. Most prominently, NF- $\kappa$ B and MAPKs are activated upon stimulation of CD95. ${ }^{16,22,31-33}$ As details of these processes remain unknown, the underlying mechanisms are currently under investigation. Caspase-8 and c-FLIP molecules have been attributed crucial roles in both apoptotic and non-apoptotic signaling of CD95.

In this study, a first integrated kinetic model of CD95mediated apoptosis and MAPK signaling was proposed. The model together with experimental data obtained using overexpression of $C-F L I P_{L}, C-F L I P_{R}$ or downregulation of $c-F L I P$, as well as inhibition of caspases by zVAD-fmk or downregulation of caspase-8, allowed to make a number of important findings. It was demonstrated that caspase-8 activity plays the central role in CD95-mediated MAPK induction. Furthermore, it was found in silico with the following experimental confirmation that it is the ratio of c-FLIP to procaspase-8 at the DISC which plays the central role in the regulation of MAPK induction by defining the amount of active caspase-8 generated at the DISC. It has been reported before that caspase activity plays a crucial role in the induction of the MAPK pathway. ${ }^{17,21}$ In our study, we defined that this caspase activity is attributed to the DISC-derived caspase-8 activity and quantitatively analyzed the contribution of DISC dynamics to MAPK activation.

$c-F L I P_{L}$ and $c-F L I P_{R}$ were shown to have different functions in CD95 stimulation. C-FLIP $P_{\mathrm{R}}$ acts as an antiapoptotic regulator at the DISC and blocks the activation of procaspase- $8 .{ }^{1}$ In contrast, the relative cellular concentration of $c-F L I P_{L}$ in part determines whether c-FLIP $P_{L}$ exerts a pro- or antiapoptotic function. Although overexpression of $c-F L I P L$ inhibits activation of procaspase-8, lower concentrations of C-FLIP $P_{L}$ can promote cleavage of procaspase- $8 .{ }^{30}$ Consistent with these data, overexpression of $c-F_{L} P_{R}$ or $C-F L I P L$ in HeLa-CD95 cells inhibited the generation of caspase-8 cleavage products in this work. Moreover, cell death occurred to a lesser extent in HeLa-CD95- $F_{R}$ or HeLa-CD95- $F_{L}$ cells compared to HeLa-CD95 cells.

Both C-FLIP downregulation and overexpression were demonstrated to block procaspase-8 processing and MAPK activation in response to anti-APO-1 stimulation. Procaspase8 is processed more rapidly in C-FLIP $\mathrm{L}_{\mathrm{L}}$-procaspase-8 heterodimers than in procaspase- 8 homodimers. ${ }^{30}$ Thus, C-FLIPL can have a catalytic effect on procaspase-8 processing, that is, its downregulation can reduce procaspase-8 activation. This was observed in our experiments and further confirmed using modeling.

A mechanism has been proposed in which catalytic activity and substrate specificity of caspase- 8 are determined by conformation and cleavage status of procaspase- $8 .{ }^{8}$ Specifically, it was suggested that the substrate repertoire of procaspase-8 is restricted to itself and c-FLIP, whereas the active $\mathrm{p} 43 / \mathrm{p} 41-\mathrm{p} 10$ exhibits a broader substrate repertoire, including procaspase-3 and Bid. In this work, procaspase-8 processing is required for CD95-induced activation of MAPKs, as the conditions impairing MAPK activation are accompanied by reduced procaspase- 8 processing. Both the first and the second cleavage step are in part affected, pointing out the potential role of $\mathrm{p} 43 / \mathrm{p} 41$ and $\mathrm{p} 18$ in MAPK activation. The substrate of caspase-8 activity leading to MAPK activation has to be elucidated in the future. In addition, procaspase-10, the second initiator caspase at the DISC, might also contribute 
to CD95-mediated MAPK activation. It was reported before that it could not substitute caspase- 8 in its apoptotic function. ${ }^{6}$ However, the contribution of procaspase-10 to the induction of non-apoptotic pathways remains to be addressed.

In our modeling approach, we concentrated on early signaling events, which occurred within a few hours after CD95 stimulation. It will be a future challenge to combine a model of CD95-mediated signal transduction with a model of transcriptional regulation to understand the possible feedback from transcriptional regulation by MAPKs on upstream CD95 signaling. In addition, a combination of the CD95-mediated MAPK model with our recent model of the crosstalk between CD95-mediated NF- $\mathrm{B}$ induction and apoptosis ${ }^{16}$ would provide a more generic view on the mechanism of life/death decisions at CD95.

Our results support the new paradigm in CD95 signaling that the CD95 DISC can act as a main switch deciding between life and death. ${ }^{20}$ Furthermore, we have shown in quantitative terms that the balance between C-FLIP and procaspase- 8 regulates the onset of MAPK activation. Interestingly, dynamics of C-FLIP and procaspase- 8 at the DISC is also a central regulatory point for NF- $\kappa$ B induction and apoptosis. These regulatory mechanisms provide an important basis for understanding the crosstalk between nonapoptotic and apoptotic signaling pathways. This knowledge will provide new insights into multiple diseases, in particular with regard to the sensitivity and resistance of cancer cells towards apoptosis, as modulation of the ratio between procaspase-8 and c-FLIP in cancer cells might influence their resistance towards apoptosis.

\section{Materials and Methods}

Cell lines. HeLa-CD95 was generated by selection with $20 \mathrm{ng} / \mathrm{ml}$ puromycin according to standard protocols. ${ }^{30}$ Stable $c-F L I P_{L}$ and $c-F L I P_{R}$ cell lines were selected with $100 \mu \mathrm{g} / \mathrm{ml} \mathrm{G} 418$ and $100 \mu \mathrm{g} / \mathrm{ml}$ zeocin, respectively. ${ }^{30} \mathrm{HeLa}-\mathrm{CD} 95$ cells were maintained in DMEM (Life Technologies, Germany), 10 mM HEPES (Life Technologies), $50 \mu \mathrm{g} / \mathrm{ml}$ gentamycin (Life Technologies), and 10\% fetal calf serum (Life Technologies) in $5 \% \mathrm{CO}_{2}$. Transfections were carried out using Lipofectamine 2000 (Invitrogen)

Antibodies and reagents. Anti-caspase- 8 monoclonal antibody $\mathrm{C} 15$ (mouse IgG2b) recognizes the p18 subunit of caspase-8. ${ }^{5}$ Anti-FLIP monoclonal antibody NF6 (mouse lgG1) recognizes the N-terminal part of c-FLIP. ${ }^{12}$ Anti-FADD monoclonal antibody 1C4 (mouse lgG1) recognizes the C-terminal part of FADD. Anti-APO-1 (anti-CD95) is an agonistic monoclonal antibody (IgG3) recognizing an epitope on the extracellular part of CD95 (APO-1/Fas). In addition, we used C2O rabbit polyclonal CD95 antibody (Santa Cruz Biotechnology, Heidelberg, Germany), anti-p38 monoclonal antibody 5F11 (mouse IgG2b; Cell Signaling Technology, Frankfurt/Main, Germany), anti-phospho-p38 rabbit polyclonal (Promega, Mannheim, Germany), anti-phospho-Erk monoclonal antibody (mouse IgG2a; Santa Cruz Biotechnology), anti-Erk monoclonal antibody (mouse lgG1; BD Signal Transduction, Heidelberg, Germany), and anti-tubulin monoclonal antibody (mouse IgG1; Sigma-Aldrich Steinheim, Germany). Horseradish peroxidase-conjugated goat anti-mouse IgG1, -2a and -2b were from Southern Biotechnology Associates (Echingen, Germany). All chemicals used were of analytical grade and purchased from Merck (Darmstadt, Germany) or Sigma-Aldrich (Steinheim, Germany).

Analysis of total cellular lysates. A total of $1 \times 10^{7}$ cells were either treated with indicated amounts of anti-CD95 for indicated periods of time at $37^{\circ} \mathrm{C}$ or left untreated, washed twice in $1 \times$ PBS and lysed subsequently in lysis buffer ( $30 \mathrm{mM}$ Tris/ $\mathrm{HCl}, \mathrm{pH} 7.5,150 \mathrm{mM} \mathrm{NaCl}, 2 \mathrm{mM}$ EDTA, $1 \mathrm{mM}$ phenylmethylsulfonyl fluoride (Sigma), protease inhibitor cocktail, 1\% Triton X-100 (Serva, Germany), and $10 \%$ glycerol). If pretreated with ZVAD-fmk, cells were pre-incubated for $30 \mathrm{~min}$ at $37^{\circ} \mathrm{C}$ with the indicated concentrations of ZVAD-fmk before stimulation. Total cellular lysates were analyzed using SDS-PAGE gels. Proteins were transferred to a Hybond nitrocellulose membrane (Amersham Pharmacia Biotech, Freiburg, Germany), blocked with $5 \%$ non-fat dry milk in PBS/Tween (PBS with $0.05 \%$ Tween-20) for $1 \mathrm{~h}$, washed with PBS/Tween, and incubated with the primary antibody in PBS/Tween overnight at $4{ }^{\circ} \mathrm{C}$. For all phospho-specific antibodies TBS/Tween (TBS with $0.05 \%$ Tween-20) was used. Blots were developed with a chemoluminescence method according to the manufacturer's protocol (PerkinElmer Life Sciences, Rodgau, Germany).

Live cell imaging. Cells were cultured in eight-well Lab-Tek-chambered cover glasses (Nunc, Langenselbold, Germany). During imaging, cells were maintained at $37^{\circ} \mathrm{C}$ in DMEM without phenol red and complemented with $20 \mathrm{mM}$ HEPES buffer and 10\% fetal calf serum. We used a Leica SP2 laser scanning confocal microscope (Leica Microsystems, Wetzlar, Germany) with a $\times 63$ oil immersion lens.

CD95 DISC immunoprecipitation. A total of $1 \times 10^{7}$ HeLa-CD95 cells were lysed in lysis buffer, without or with anti-APO-1 stimulation, divided into two equal parts and the DISC immunoprecipitation was performed by using $5 \mu \mathrm{g}$ anti-CD95 (anti-APO-1) antibody together with $30 \mu \mathrm{l}$ of Protein-A-Sepharose. Immunoprecipitations were performed overnight at $4^{\circ} \mathrm{C}$, and then beads were washed five times with 20 volumes of lysis buffer and subjected to western blot analysis as described above.

Stable siRNA expression. We used stable expression of siRNA as published previously. ${ }^{34}$ The siRNA FLIP vector or control empty vector were transfected into the HeLa-CD95 cell line as described earlier. The knockdown of caspase-8 was carried out with a pSilencer 3.1-H1 Neo vector. The siRNA target sequence was $5^{\prime}$-GGGTCATGCTCTATCAGAT- $3^{\prime}$ as described. ${ }^{35}$

Quantification of western blot time series. The western blots were developed using detection with a CCD camera or scanned and analyzed with ImageJ (http://rsb.info.nih.gov/ij/). The local background from the bands was subtracted and their intensity was quantified. For normalization, the signal was divided by the anti-tubulin signal. For alignment of time series from repeated experiments, the signals were multiplied with a scalar to minimize the sum of meansquared distances of measurements at the same time points. Finally, the average signal was computed and scaled.

Modeling and simulation. Model fitting and simulation was carried out using the Matlab toolbox PottersWheel (Natick, MA, USA). ${ }^{36}$ The model was modified from our previous publication. ${ }^{30}$ The differential equations, kinetic parameters and the details of the fitting procedure can be found in the Supplementary information.

\section{Conflict of Interest}

The authors declare no conflict of interest

Acknowledgements. We thank Selcen Öztürk for critical comments on the manuscript. We acknowledge support by the Helmholtz Alliance on Systems Biology (NW1SBCancer/UniHD), the BMBF funded ForSys centre VIROQUANT (0313923), the BMBF funded Virtual Liver project, Helmholtz-Russia Joint Research Groups2008-2, and Helmholtz Alliance Immunotherapy of Cancer (HA-202) for supporting our work.

1. Krammer PH, Arnold R, Lavrik IN. Life and death in peripheral T cells. Nat Rev Immunol 2007; 7: 532-542.

2. Peter ME, Budd RC, Desbarats J, Hedrick SM, Hueber AO, Newell MK et al. The CD95 receptor: apoptosis revisited. Cell 2007; 129: 447-450.

3. Kischkel FC, Hellbardt S, Behrmann I, Germer M, Pawlita M, Krammer PH et al. Cytotoxicity-dependent APO-1 (Fas/CD95)-associated proteins form a death-inducing signaling complex (DISC) with the receptor. EMBO J 1995; 14: 5579-5588.

4. Scott FL, Stec B, Pop C, Dobaczewska MK, Lee JJ, Monosov E et al. The Fas-FADD death domain complex structure unravels signalling by receptor clustering. Nature 2009; 457: 1019-1022.

5. Scaffidi C, Medema JP, Krammer PH, Peter ME. FLICE is predominantly expressed as two functionally active isoforms, caspase-8/a and caspase-8/b. J Biol Chem 1997; 272: 26953-26958. 
6. Sprick M, Rieser E, Stahl H, Grosse-Wilde A, Weigand M, Walczak H. Caspase-10 is recruited to and activated at the native TRAIL and CD95 death-inducing signalling complexes in a FADD-dependent manner but can not functionally substitute caspase-8. EMBO J 2002; 21: 4520-4530.

7. Chang DW, Xing Z, Capacio VL, Peter ME, Yang X. Interdimer processing mechanism of procaspase-8 activation. EMBO J 2003; 22: 4132-4142.

8. Hughes MA, Harper N, Butterworth M, Cain K, Cohen GM, MacFarlane M. Reconstitution of the death-inducing signaling complex reveals a substrate switch that determines CD95mediated death or survival. Mol Cell 2009; 35: 265-279.

9. Hoffmann JC, Pappa A, Krammer PH, Lavrik IN. A new C-terminal cleavage product of procaspase-8, p30, defines an alternative pathway of procaspase-8 activation. Mol Cell Biol 2009; 29: 4431-4440.

10. Chang DW, Xing Z, Pan Y, Algeciras-Schimnich A, Barnhart BC, Yaish-Ohad S et al. $c-F L I P(L)$ is a dual function regulator for caspase-8 activation and CD95-mediated apoptosis. EMBO J 2002; 21: 3704-3714.

11. Lavrik I, Krueger A, Schmitz I, Baumann S, Weyd H, Krammer PH et al. The active caspase-8 heterotetramer is formed at the CD95 DISC. Cell Death Differ 2003; 10: 144-145.

12. Scaffidi C, Schmitz I, Krammer PH, Peter ME. The role of C-FLIP in modulation of CD95-induced apoptosis. J Biol Chem 1999; 274: 1541-1548.

13. Golks A, Brenner D, Fritsch C, Krammer PH, Lavrik IN. c-FLIPR, a new regulator of death receptor-induced apoptosis. J Biol Chem 2005; 280: 14507-14513.

14. Golks A, Brenner D, Krammer PH, Lavrik IN. The c-FLIP-NH2 terminus (p22-FLIP) induces NF-kappa B activation. J Exp Med 2006; 203: 1295-1305.

15. Ueffing N, Keil E, Freund C, Kuhne R, Schulze-Osthoff K, Schmitz I. Mutational analyses of c-FLIPR, the only murine short FLIP isoform, reveal requirements for DISC recruitment. Cell Death Differ 2008; 15: 773-782.

16. Neumann L, Pforr C, Beaudouin J, Pappa A, Fricker N, Krammer PH et al. Dynamics within the CD95 death-inducing signaling complex decide life and death of cells. Mol Syst Biol 2010; 6: 352.

17. Nakajima A, Komazawa-Sakon S, Takekawa M, Sasazuki T, Yeh WC, Yagita H et al. An antiapoptotic protein, C-FLIPL, directly binds to MKK7 and inhibits the JNK pathway. EMBO J 2006; 25: 5549-5559.

18. Barnhart BC, Legembre P, Pietras E, Bubici C, Franzoso G, Peter ME. CD95 ligand induces motility and invasiveness of apoptosis-resistant tumor cells. EMBO J 2004; 23 3175-3185

19. Kreuz S, Siegmund D, Rumpf JJ, Samel D, Leverkus M, Janssen O et al. NF kappa B activation by Fas is mediated through FADD, caspase-8, and RIP and is inhibited by FLIP. J Cell Biol 2004; 166: 369-380.

20. Lavrik IN, Golks A, Riess D, Bentele M, Eils R, Krammer PH. Analysis of CD95 threshold signaling: triggering of CD95 (FAS/APO-1) at low concentrations primarily results in survival signaling. J Biol Chem 2007; 282: 13664-13671.

21. Nakajima A, Kojima Y, Nakayama M, Yagita H, Okumura K, Nakano H. Downregulation of c-FLIP promotes caspase-dependent JNK activation and reactive oxygen species accumulation in tumor cells. Oncogene 2008; 27: 76-84.

22. Desbarats J, Birge RB, Mimouni-Rongy M, Weinstein DE, Palerme JS, Newell MK. Fas engagement induces neurite growth through ERK activation and p35 upregulation. Nat Cell Biol 2003; 5: 118-125.
23. Holmstrom TH, Schmitz I, Soderstrom TS, Poukkula M, Johnson VL, Chow SC et al. MAPK/ERK signaling in activated $T$ cells inhibits CD95/Fas-mediated apoptosis downstream of DISC assembly. EMBO J 2000; 19: 5418-5428.

24. Juo P, Kuo CJ, Reynolds SE, Konz RF, Raingeaud J, Davis RJ et al. Fas activation of the p38 mitogen-activated protein kinase signalling pathway requires ICE/CED-3 family proteases. Mol Cell Biol 1997; 17: 24-35.

25. Eissing T, Conzelmann H, Gilles ED, Allgower F, Bullinger E, Scheurich P. Bistability analyses of a caspase activation model for receptor-induced apoptosis. J Biol Chem 2004; 279: 36892-36897.

26. Legewie $\mathrm{S}$, Bluthgen $\mathrm{N}$, Herzel $\mathrm{H}$. Mathematical modeling identifies inhibitors of apoptosis as mediators of positive feedback and bistability. PLoS Comput Biol 2006; 2: e120.

27. Rehm M, Huber HJ, Dussmann H, Prehn JHM. Systems analysis of effector caspase activation and its control by X-linked inhibitor of apoptosis protein. EMBO J 2006; 25: 4338-4349.

28. Bentele M, Lavrik I, Ulrich M, Stosser S, Heermann DW, Kalthoff $\mathrm{H}$ et al. Mathematical modeling reveals threshold mechanism in CD95-induced apoptosis. J Cell Biol 2004; 166 839-851.

29. Spencer SL, Gaudet S, Albeck JG, Burke JM, Sorger PK. Non-genetic origins of cell-to-cell variability in TRAIL-induced apoptosis. Nature 2009; 459: 428-432.

30. Fricker N, Beaudouin J, Richter P, Eils R, Krammer PH, Lavrik IN. Model-based dissection of CD95 signaling dynamics reveals both a pro- and antiapoptotic role of c-FLIPL. J Cell Biol 2010; 190: 377-389.

31. Kataoka T, Budd RC, Holler N, Thome M, Martinon F, Irmler M et al. The caspase-8 inhibitor FLIP promotes activation of NF-kappaB and Erk signaling pathways. Curr Biol 2000; 10: 640-648.

32. Ahn JH, Park SM, Cho HS, Lee MS, Yoon JB, Vilcek J et al. Non-apoptotic signaling pathways activated by soluble Fas ligand in serum-starved human fibroblasts Mitogen-activated protein kinases and NF-kappaB-dependent gene expression. J Biol Chem 2001; 276: 47100-47106

33. Wajant H, Pfizenmaier K, Scheurich P. Non-apoptotic Fas signaling. Cytokine Growth Factor Rev 2003; 14: 53-66.

34. Diessenbacher P, Hupe M, Sprick MR, Kerstan A, Geserick P, Haas TL et al. NF-kappaB inhibition reveals differential mechanisms of TNF versus TRAIL-induced apoptosis upstream or at the level of caspase-8 activation independent of cIAP2. J Invest Dermatol 2008; 128: 1134-1147.

35. Wagner KW, Engels IH, Deveraux QL. Caspase-2 can function upstream of bid cleavage in the TRAIL apoptosis pathway. J Biol Chem 2004; 279: 35047-35052.

36. Maiwald T, Kreutz C, Pfeifer AC, Bohl S, Klingmuller U, Timmer J. Dynamic pathway modeling: feasibility analysis and optimal experimental design. Ann NY Acad Sci 2007 1115: 212-220.

\section{(c)}

Cell Death and Disease is an open-access journal published by Nature Publishing Group. This work is licensed under the Creative Commons Attribution-Noncommercial-Share Alike 3.0 Unported License. To view a copy of this license, visit http:// creativecommons.org/licenses/by-nc-sa/3.0/

Supplementary Information accompanies the paper on Cell Death and Disease website (http://www.nature.com/cddis) 